\title{
Pediatric ventricular assist device support as a permanent therapy: Clinical reality
}

\author{
Iki Adachi, MD
}

\footnotetext{
From Michael E. DeBakey Department of Surgery, Congenital Heart Surgery, Texas Children's Hospital, Baylor College of Medicine, Houston, Tex.

Received for publication Jan 5, 2019; revisions received Feb 15, 2019; accepted for publication Feb 22, 2019. Address for reprints: Iki Adachi, MD, Michael E. DeBakey Department of Surgery, Congenital Heart Surgery, Texas Children's Hospital, Baylor College of Medicine, 6651 S. Main St, 19345H, Houston, TX 77030 (E-mail: iadachi@bcm.edu).

J Thorac Cardiovasc Surg 2019;158:1438-41

$0022-5223 / \$ 36.00$

Copyright (c) 2019 by The American Association for Thoracic Surgery

https://doi.org/10.1016/j.jtcvs.2019.02.145
}

Feature Editor's Note-Dr Adachi, in this Expert Opinion piece, introduces us to a paradigm shift in the management of pediatric patients with end-stage heart failure. The group at Texas Children's Hospital appears to be on the leading edge of this curve, given their extensive experience with ventricular assist devices (VADs). The more favorable risk profile of continuous-flow VADs compared with pulsatile VADs has led to their institutional policy of waiting 3 months following VAD implantation until listing for heart transplantation. This policy appears to have been quite effective and has actually been in effect since 2012. This "no-rush" strategy is enabled by the decreased morbidity with the continuous-flow pump, which maximizes the opportunity for the recuperation of the patient not only physically but psychologically before the transplant.

Dr Adachi then piques our interest by discussing the concept of VAD support as a permanent therapy for pediatric patients. He emphasizes the strengths of this approach, which include unrestricted accessibility, durability, manageability, and continual technological advancement. With further improvement in VAD technology, the current strategy of pediatric cardiac transplantation as an exit strategy from VAD support may evolve into a role as a bridge to permanent VAD support (total artificial heart). In the (near?) future, permanent pediatric VAD support may become the new reality. This will also be an opportunity for long-term support of patients who are not candidates for transplant either based on religious beliefs, elevated panel reactive antibody, elevated pulmonary vascular resistance, or other confounding factors.

Congratulations to Dr Adachi and the Heart Center Team at Texas Children's Hospital on continuing to move us in new and improved directions for the optimal, strategic use of VADs in the management of children with end-stage heart failure.

Carl L. Backer, MD

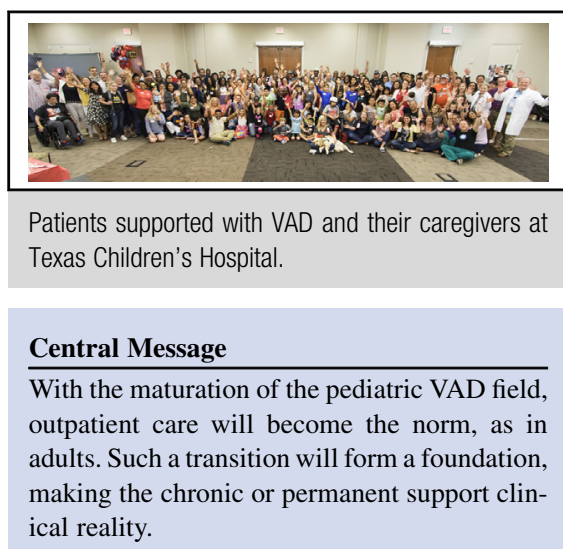

This Invited Expert Opinion provides a perspective on the following paper: Journal of the American College of Cardiology. 2018: 72:402-415. https://doi.org/10.1016/j.jacc.2018 04.072 .

See Commentaries on pages 1442 and 1444 .

The field of pediatric ventricular assist device (VAD) support has grown exponentially over the last decade, the birth of which was the introduction of a pediatric-specific pulsatile VAD, ie, EXCOR (Berlin Heart, Inc, The Woodlands, Tex). ${ }^{1}$ With increasing exposure to VAD support, pediatric centers have recognized the superiority of VAD physiology over extracorporeal membrane oxygenation, which had long been the mainstay of pediatric mechanical circulatory support. This experience has formed a solid foundation, allowing the practice of pediatric VAD support to move into the next phase: the introduction of implantable, continuous-flow VADs. Having faced the challenges inherent to pulsatile VAD, such as devastating neurologic events, the pediatric field has enthusiastically adopted the use of continuous-flow VAD, particularly miniaturized pumps such as HeartWare HVAD (Medtronic, Inc, Minneapolis, Minn). ${ }^{2,3}$

Despite booming experiences, the philosophy underlying the management of implantable, continuous-flow VAD in the pediatric population remains fundamentally different than that in the adult population. As demonstrated by the pediatric HVAD experience in the Pedimacs registry, ${ }^{3}$ as well as in a worldwide survey, ${ }^{2}$ approximately one half of pediatric patients with HVAD are managed as inpatients, which 
is in stark contrast to the fact that outpatient management is standard practice in the adult population. Support duration is also remarkably different among the groups. At 3 months after continuous-flow VAD implant, approximately one half of pediatric patients are already transplanted, whereas the vast majority $(95 \%)$ of adult patients remain on VAD. ${ }^{4}$

The reasons for such a notable difference are multifactorial. Children having better access to donor hearts is one of the reasons. Another reason, which is certainly modifiable, is the mindset of pediatric physicians; currently, continuous-flow VAD is managed identically to pulsatile VAD, despite the latter having a more significant complication profile. Since the singular focus is avoidance of VADrelated complications, many pediatric patients on continuous-flow VAD support are kept in the hospital, committed to imminent cardiac transplantation. As soon as VAD support is commenced, the child and the responsible physician tag-team in a race against the complications clock to locate a viable heart to cross the bridge to transplant. All things considered, the "rushing approach" of the current management strategy is driven by a sense of urgency, resulting in anticipative transplantation.

Undeniably, getting a child through VAD support without complications is considered by many, if not all, pediatric physicians as the best-case scenario. Determining the optimal timing of VAD explant (typically with transplant) is predicated upon an interplay of several parameters, including patient and institutional factors, all of which must be appropriately weighed in a risk/benefit analysis. Yet, it is my opinion that such a rushing approach is the result of an imbalance in the risk/benefit analysis of VAD support by overemphasizing the benefit of minimizing the support duration above all, regardless of VAD type. This is despite the fact that virtually all clinicians agree that continuousflow VAD support carries a more favorable risk profile than pulsatile VAD. In light of this and other recent progress, I submit that the time has come for selected factors in the decision-making process to be redefined and reevaluated.

Foremost, a thoroughly informed decision cannot be made without fully recognizing the benefits of a moredeliberate ("no-rush") approach. Heart failure in a child significantly impacts both the patients and their families. A vast majority of pediatric patients under consideration for VAD are profoundly frail before and in the early days of support. Therefore, dedicating a certain period of time permitting the child to adapt to their new life with a VAD (at least a couple of months depending on the preoperative status; the sicker preoperatively, the longer time necessary postoperatively) before cardiac transplant maximizes the opportunity for recuperation, not only physically but psychologically. ${ }^{6}$ Improved cardiac output with VAD support allows nutritional resuscitation as well as physical rehabilitation. In addition, home discharge permits psychological recovery from traumatic experiences in the hospital.
Staying at home with family and socializing with friends at school are crucial for children to regain a sense of selfdetermination. Moreover, the importance of these advantages as they relate to familial dynamics should not be underestimated; this dedicated period allows family members to acquaint themselves with a "new normal" of independent device management and round-the-clock care.

Besides these patient factors, there are some benefits of "no rush" into transplant from a surgical standpoint. In general, surgical reinterventions within a few months of the original procedure are not ideal because of mediastinal tissue inflammation and adhesion, which could increase the risk of bleeding and injury to vital structures such as the phrenic nerves. Lastly, and most importantly, waiting a certain period on VAD provides an opportunity to assess for recovery of native cardiac function. As demonstrated by studies in the adult population, the younger the patient, the greater the recovery potential. ${ }^{7,8}$ Nevertheless, the actual recovery rate leading to VAD explantation is lower in the Pedimacs registry $(0.6 \%)$ than in the INTERMACS registry $(1 \%){ }^{5}$ Of all the factors that may explain this counterintuitive phenomenon, the current "rushing approach" is strongly implicated. As alluded to earlier, approximately $50 \%$ of children with implantable, continuous-flow VAD support undergo cardiac transplantation within 3 months of VAD implant. Given the anecdotal wisdom that failing hearts need at least a couple of months of rest on VAD to begin showing signs of functional recovery, ${ }^{9}$ it is possible that potentially recoverable hearts are replaced prematurely. In the absence of tests that reliably predict the potential for cardiac recovery, the only way to establish the irreversibility of heart failure is by allowing sufficient time to facilitate repeated observation of an adequately decompressed heart on VAD support.

Taking these considerations in mind, we introduced a 3month waiting policy after continuous-flow VAD implant in 2012 at Texas Children's Hospital. ${ }^{4}$ With this policy, patients are kept inactivated on the transplant wait-list (if already on the list) for at least 3 months, unless there are significant concerns with long-term VAD support. In our experience of $>50$ HVAD implants to date, most patients tolerate the waiting period well without major issues, as evidenced by a 1-year survival rate of $97 \%$. Only a few patients required activation on the transplant list before the 3month mark. Although none of the pediatric centers in North America have currently adopted a similar policy, some institutions are now moving away from the rushing approach toward a more deliberate approach, ie, longterm support akin to that in adults. With the maturation of pediatric VAD field, it is anticipated that true long-term support will become the rule rather than the exception. Feasibility of providing true long-term support has the potential to drastically change the outlook of pediatric heart failure management. 
In principle, pediatric cardiac transplantation is a timelimited palliation, primarily due to limited cardiac graft durability, with 10-year graft survival approximated at $70 \% .{ }^{10}$ Granted that cardiac transplantation represents the only practical life-saving procedure in many circumstances, the limited organ durability is unlikely to offer a normal life expectancy. The discrepancy between "natural" versus "transplant recipient" life expectancies is more substantial in the pediatric population compared with that of adults, given the earlier age at transplant in the pediatric group. Theoretically, repeat transplantation could be offered to offset the limited durability of the primary organ. It is known, however, the graft durability after repeat cardiac transplantation is generally inferior to the primary transplant. $^{11}$ In our experience at Texas Children's Hospital, the 10-year graft survival after repeat cardiac transplantation is approximately $30 \%$, as opposed to approximately $70 \%$ for primary cardiac transplantation. Given the worse graft durability in the setting of severe organ shortage, offering repeat cardiac transplantation presents an ethical challenge. ${ }^{12,13}$ What could be a potential solution to address the limited life expectancies in pediatric patients with end-stage heart failure, even after a successful cardiac transplant, which is currently considered to be the last resort in the management of pediatric heart failure?

VAD support as a permanent therapy may become the solution. In my opinion, the combination of mechanical circulatory support and cardiac transplantation is an option to bridge the gap in life expectancies. The strengths and weaknesses of each modality are discussed to follow. Several important features of VAD are definitely superior to cardiac transplantation. First, access to the pump (ie, accessibility) is unrestricted as a nature of industrial products, which is in stark contrast to the stagnant organ supply of cardiac allografts. ${ }^{10}$ Second, longevity of pump function (ie, durability) will likely surpass that of cardiac graft in the near future. Ease of medication control (ie, manageability) is, in general, better with VAD than with transplant, primarily due to not needing immunosuppressants, even taking the need for anticoagulation into account for the former group. Last, and perhaps, most importantly, the room for further improvement (ie, advanceability) is substantially greater in VAD technology. Although there has been a significant improvement in VAD outcomes over the last decade, virtually no improvement has been observed in the posttransplant outcomes since the introduction of cyclosporine in 1984 except early surgical survival. ${ }^{14}$ The VAD field would further advance in many aspects, including novel pump design (such as HeartMate $3^{15}$ and Jarvik 2015 ${ }^{16}$ ), advanced technologies (such as transcutaneous energy transmission ${ }^{17}$ ), and new monitoring systems (such as implantable pulmonary artery pressure sensor ${ }^{18}$ ).

In contrast, VAD is certainly inferior to transplant in certain aspects. Varied anatomical morphology associated with congenital cardiac anomalies, often precluding appropriate device fit, is a major limitation with VAD support (ie, adaptability). For instance, heavy myocardial trabeculation in a morphologic right ventricle, seen in failing single ventricle patients, hinders placement of a VAD's inflow cannula. In contrast, a cardiac allograft has great flexibility, which would allow for implantation in patients with complex cardiac and thoracic anatomy, such as heterotaxy. Activation of the coagulation cascade (ie, hemocompatibility) is another serious issue with the current VAD interface, which requires intensive anticoagulation and platelet inhibition. Although the tendency for thrombosis is less with continuous-flow VAD technology than pulsatile pumps, hemolysis tends to be more substantial in the former, especially in children, on account of greater pump rotational speeds and a smaller clearance area for flow path. ${ }^{19}$ Lastly, the lack of parallel increase in size (ie, growth ability) of VAD in a growing child is an obvious shortcoming.

In light of the aforementioned points, perhaps combining selected strengths of both modalities into the treatment roadmap for each patient may represent the solution to fill the gap in life expectancies. More specifically, the role of a pediatric cardiac transplant will transition from being the destination to forming a basis for eventual chronic (or even permanent) VAD support, by allowing for somatic growth in small children or providing more favorable anatomical adoptability in those with complex congenitally malformed hearts. In other words, the current function of pediatric cardiac transplant as an exit strategy for VAD support (ie, bridge-to-transplant) may evolve into a new role as a bridge-to-permanent VAD support (ie, reversed-bridge). Such future direction is not beyond the scope of imagination, given the rapid maturation of the pediatric VAD support in the recent years. There are several promising devices on the horizon for that particular purpose. ${ }^{20,21}$ Battery technology is also advancing expeditiously, owing to the recent rise in electric automobiles, such as Tesla (Palo Alto, Calif). A fully charged battery in the Tesla Model $\mathrm{S}$ has the capability of running the HVAD pump for $>365$ days without recharging. The creation of a small battery that can run an implantable VAD just for a couple of days may not be technically impossible.

As we recently demonstrated in our article, ${ }^{4}$ pediatric implantable VAD outpatient management is feasible even in small children with complex congenital cardiac malformation. One of our patients with HVAD support is now supported for more than 7 years (still ongoing). The other patient with HVAD for failing Fontan ( $>3$ years of support) has recently married and now expecting the arrival of their first child. Pediatric chronic VAD support is definitely a reality. The biggest obstacle to a more widespread adoption of this approach remains the mindset of pediatric clinicians who view cardiac transplantation as reaching the summit of the mountain, when in all likelihood it represents a false 
peak beyond which lies an even greater challenge in the form of chronic graft failure. As pediatric clinicians gain more experience and confidence in the management of chronic support with implantable VADs, there will be a recognition of "no need for rushing." With the maturation of the pediatric field, chronic outpatient support will become the norm, as in the adult field. The cusp of such a transition is now coming into view. Completion of the transition will then represent the beginning of the paradigm change, which is characterized by the use of implantable VAD technology as a permanent therapy.

\section{Conflict of Interest Statement}

Dr Adachi serves as consultant/proctor for Berlin Heart Inc, Medtronic Inc, Jarvik Inc, BiVACOR Inc, and consultant for Sony-Olympus Medical Solutions Inc.

\section{References}

1. Fraser CD, Jaquiss RDB, Rosenthal DN, Humpl T, Canter CE, Blackstone EH, et al. Prospective trial of a pediatric ventricular assist device. $N$ Engl J Med. 2012;367:532-41.

2. Conway J, Miera O, Adachi I, Maeda K, Eghtesady P, Henderson H, et al. Worldwide experience of a durable centrifugal flow pump in pediatric patients. Semin Thorac Cardiovasc Surg. 2018;30:327-35.

3. VanderPluym CJ, Adachi I, Niebler R, Griffiths E, Fynn-Thompson F, Chen S, et al. Outcomes of children supported with an intracorporeal continuous-flow left ventricular assist system. J Heart Lung Transplant. 2019;38:385-93.

4. Adachi I, Zea-Vera R, Tunuguntla H, Denfield SW, Elias B, John R, et al. Centrifugal-flow ventricular assist device support in children: a single center experience. J Thorac Cardiovasc Surg. 2019;157:1609-17.e2.

5. Adachi I. Continuous-flow ventricular assist device support in children: a paradigm change. J Thorac Cardiovasc Surg. 2017;154:1358-61.

6. Wilson SR, Givertz MM, Stewart GC, Mudge G H. Ventricular assist devices the challenges of outpatient management. J Am Coll Cardiol. 2009;54:1647-59.

7. Topkara VK, Garan AR, Fine B, Godier-Furnemont AF, Breskin A, Cagliostro B, et al. Myocardial recovery in patients receiving contemporary left ventricular assist devices: results from the interagency registry for mechanically assisted circulatory support (INTERMACS). Circ Heart Fail. 2016;9:e003157.
8. Wever-Pinzon O, Drakos SG, McKellar SH, Horne BD, Caine WT, Kfoury AG, et al. Cardiac recovery during long-term left ventricular assist device support. $J$ Am Coll Cardiol. 2016;68:1540-53.

9. Drakos SG, Wever-Pinzon O, Selzman CH, Gilbert EM, Alharethi R, Reid BB, et al. Magnitude and time course of changes induced by continuous-flow left ventricular assist device unloading in chronic heart failure: insights into cardiac recovery. J Am Coll Cardiol. 2013;61:1985-94.

10. Rossano JW, Dipchand AI, Edwards LB, Goldfarb S, Kucheryavaya AY, Levvey BJ, et al. The registry of the international society for heart and lung transplantation: nineteenth pediatric heart transplantation report-2016; Focus theme: primary diagnostic indications for transplant. J Heart Lung Transplant. 2016;35:1185-95.

11. Rizvi SA, Luc JGY, Choi JH, Phan K, Moncho Escriva E, Patel S, et al. Outcomes and survival following heart retransplantation for cardiac allograft failure: a systematic review and meta-analysis. Ann Cardiothorac Surg. 2018 7:12-8.

12. Colburn D. The dilemma of repeat transplants. The Washington Post. November 30, 1993. Available at: https://www.washingtonpost.com/archive/lifestyle/ wellness/1993/11/30/the-dilemma-of-repeat-transplants/7c9404a4-a8ae-4f78a98d-2c39501ce3b3/?noredirect $=$ on\&utm_term $=.7 \mathrm{e} 83 \mathrm{~b} 49439 \mathrm{a} 8$. Accessed September 23, 2019.

13. Ubel PA, Arnold RM, Caplan AL. Rationing failure: the ethical lessons of the retransplantation of scarce vital organs. JAMA. 1993;270:2469-74.

14. Morales DL, Dreyer WJ, Denfield SW, Heinle JS, McKenzie ED, Graves DE et al. Over two decades of pediatric heart transplantation: how has survival changed? J Thorac Cardiovasc Surg. 2007;133:632-9.

15. Mehra MR, Naka Y, Uriel N, Goldstein DJ, Cleveland JC, Colombo PC, et al. A fully magnetically levitated circulatory pump for advanced heart failure. $N$ Engl J Med. 2017:376:440-50.

16. Adachi I, Burki S, Horne D, Costas GG, Spangler T, Jarvik R, et al. The miniaturized pediatric continuous-flow device: preclinical assessment in the chronic sheep model. J Thorac Cardiovasc Surg. 2017;154:291-300.

17. Fu Y, Hu L, Ruan X, Fu X. A transcutaneous energy transmission system for artificial heart adapting to changing impedance. Artif Organs. 2015;39:378-87.

18. Heywood JT, Jermyn R, Shavelle D, Abraham WT, Bhimaraj A, Bhatt K, et al. Impact of practice-based management of pulmonary artery pressures in 2000 patients implanted with the CardioMEMS Sensor. Circulation. 2017;135: 1509-17.

19. Chan CHH, Diab S, Moody K, Frazier OH, Sampaio LC, Fraser CD, et al. In vitro hemocompatibility evaluation of ventricular assist devices in pediatric flow conditions: a benchmark study. Artif Organs. 2018;42:1028-34.

20. Cohn WE, Timms DL, Frazier OH. Total artificial hearts: past, present, and future. Nat Rev Cardiol. 2015;12:609-17.

21. Fukamachi K, Karimov JH, Byram NA, Sunagawa G, Dessoffy R, Miyamoto T, et al. Anatomical study of the Cleveland Clinic continuous-flow total artificial heart in adult and pediatric configurations. J Artif Organs. 2018;21:383-6. 